\title{
Paternal pericentric inversion of chromosome 4 as a cause of recurrent pregnancy loss
}

\author{
G C Wolf, J Mao, L Izquierdo, G Joffe
}

\begin{abstract}
A paternal pericentric inversion of chromosome 4 was ascertained through karyotype analysis of an abortus specimen proven to be $46, X X$, rec(4), dup $q$, inv (4)(p13q28). The relationship of paternal pericentric inversion to pregnancy loss is discussed, and a recommendation for karyotype analysis of recurrent abortion specimens is made.
\end{abstract}

(f Med Genet 1994;31:153-155)

Recurrent abortion, generally referring to the consecutive loss of two or more first trimester conceptions, afflicts approximately $2 \%$ of couples. ${ }^{1}$ Though of obvious import to the individual couple so affected, the total contribution of parental chromosomal abnormalities to the aetiology of early pregnancy loss is exceedingly small, no more than $5 \%,,^{2-5}$ and possibly as low as 1 to $2 \%^{6}$; karyotypic analysis of 2500 spontaneous abortions detected only six $(0.24 \%)$ as a result of major parental anomalies. ${ }^{1}$ Pericentric inversions account for only 5 to $10 \%$ of the major chromosomal abnormalities found in couples with recurrent pregnancy loss. Hence the overall incidence of pericentric inversion as a cause of recurrent pregnancy loss is quite small $(1 / 10000)^{7}$; fewer than 20 such cases involving chromosome 4 have been reported. We describe here a couple with three consecutive first trimester losses, with the father having the karyotype $46, \mathrm{XY}$,inv(4)(p13q28).

Reproductive
Endocrinology,
Department of

Obstetrics and

Gynecology,

University of New

Mexico School of

Medicine,

Albuquerque, New

Mexico 87131 USA

G C Wolf

$\mathrm{J}$ Mao

Division of Maternal

Fetal Medicine,

Department of

Obstetrics and

Gynecology,

University of $\mathrm{New}$

Mexico School of

Medicine,

Albuquerque, New

Mexico 87131 USA

L Izquierdo

G Joffe

Correspondence to Dr Wolf, Department of Obstetrics and Gynecology, University of South Carolina School of Medicine, Two Richland Medical Park, Suite 208, Columbia, SC 29203, USA.

Received 2 July 1993

Revised version accepted for publication 18 August 1993

\section{Case report}

with the onset of her fourth pregnancy. conception occurred at the age of without complication. At the age of 35 , after 12 months of attempting conception, she achieved a pregnancy with a different (and partner. That pregnancy neously aborted at 8 weeks' gestation. Over th two years she continued attempts ultimately conceiving and taneously aborting a third pregnancy at ap- proximately 7 weeks' gestation. Her fourth conception occurred four months later, while being treated with clomiphene citrate, at the age of 38. An ultrasound examination at 7 weeks' gestation confirmed fetal cardiac activity; at 10 weeks she noted vaginal bleeding and a repeat ultrasound examination showed a non-viable pregnancy. A dilatation and curettage was performed and a karyotype was obtained on the products of conception and, subsequently, on the parents.

The maternal karyotype was $46, \mathrm{XX}$, the paternal karyotype was $46, X Y, \operatorname{inv}(4)(\mathrm{p} 13 \mathrm{q} 28)$, and the fetal analysis showed the recombinant $46, X X, \operatorname{rec}(4)$, dup q,inv(4)(p13q28)pat (fig 1). The carrier's father died at the age of 57 of lung cancer; the mother and younger brother declined karyotype analysis. A second brother proved also to be an inversion carrier; he and his wife had previously had a spontaneous first trimester loss, though a current pregnancy now at 5 months' gestation was proven karytopically normal by amniocentesis.

\section{Discussion}

Though pericentric inversions have been detected in all chromosomes with varying frequency, chromosomes $2,5,7,9$, and 10 are statistically more prone to such rearrangements. ${ }^{2}$ As many as $1 / 100$ people exhibit such an inversion in the heterochromatic region of chromosome 9 , which is felt to be simply a population variant; similar, but less frequent, inversions are found in chromosome 1 and in the $\mathrm{Y}$ chromosome. The majority of pericentric inversions are detected while investigating congenital malformations or mental retardation, and not because of recurrent pregnancy loss. Two recent investigations found 44 cases in 33442 subjects, and six in 4272 subjects studied for recurrent loss. ${ }^{23}$ A 1989 summary of several years of European, Canadian, and American prenatal diagnoses from 148 laboratories detailed 173 cases of pericentric inversions (from a total of 1833 parental abnormalities) of which only nine involved chromosome $4 .{ }^{8}$ No other pericentric inversions of chromosome 4 with breakpoints identical to the current case have been reported, though two closely related

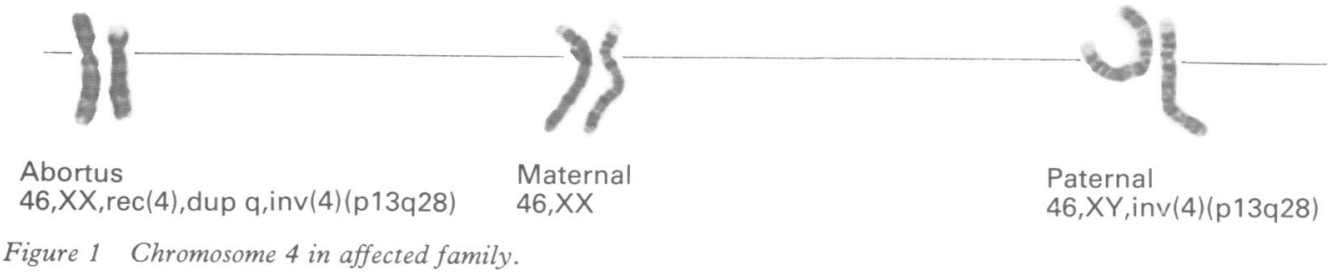


instances have been characterised, $(\mathrm{p} 12 \mathrm{q} 25)^{9}$ and (p12q26). ${ }^{10}$

Counselling couples carrying pericentric inversions regarding pregnancy outcome is difficult; an average figure for producing a liveborn abnormal offspring has generally been estimated to be 5 to $10 \%,,^{11}$ but, in fact, may be much higher or lower depending on the specific inversion. In a review of the familial data in 24 pericentric inversion index patients, most of which were ascertained through karyotyping because of mental retardation or congenital malformations, only 10 first trimester abortions in a total of 73 pregnancies $(13.7 \%)$ were noted. ${ }^{7}$ Similarly, a five generation pedigree of $24 \operatorname{inv}(4)$ (p16q21) carriers showed no statistically significant difference in the abortion rate compared to the non-carrier sibs, and no recombinant offspring were produced. ${ }^{12}$ Similarly, the abortion rate was not increased in two unrelated families involving $\operatorname{inv}(4)(\mathrm{p} 15 \mathrm{q} 11)^{13}$ and in a large kindred with $\operatorname{inv}(3)(\mathrm{p} 12 \mathrm{q} 24) .^{14}$

The mode of ascertainment of the proband obviously has a profound effect on the risk of recurrent pregnancy loss. If ascertained through repeated loss the abortion rate is over $90 \%{ }^{4}$; if through alternate modes (that is, advanced maternal age, mental retardation, congenital anomalies, routine screening) there appears to be no significantly increased risk. In fact, ascertainment through repeated pregnancy loss occurs in the minority of cases; in a series of 55 pericentric inversion cases, only four $(7 \cdot 2 \%)$ were detected secondary to recurrent pregnancy loss. ${ }^{15}$ Our method of ascertainment through analysis of the abortion specimen is exceptional.

The risk of abnormal pregnancy outcome in a pericentric inversion carrier is a consequence of the formation of gametes with partial dupli- cation and partial deletion, which in turn result from a crossover occurring within the inverted segment during meiosis. The large inverted segment usually forms a loop to pair with its (non-inverted) homologue during meiosis; if crossover occurs within the inversion, "aneusomie de recombinaison"16 results in two unbalanced gametes, one with a duplication of the inverted long arm material and a deletion of the short arm chromatin, and the other with duplication of the short arm material and a deletion of the long arm chromatin (fig 2). Chiasma formation is dependent both on the specific chromosome involved as well as on the length of the inversion. For the normal chromosome 4 the average chiasma frequency has been estimated at $2 \cdot 8^{17}$; it may be significantly different when an inversion is present.

The larger the inversion the greater the chance of crossover, but the amount of chromatin deleted will be decreased, thereby giving rise to less severely unbalanced gametes and an increased chance of a liveborn, unbalanced offspring. Alternatively, as the inversion becomes smaller there is less chance of chiasma formation, both because of decreased length, but also because of the known diminished incidence of chiasma formation near the centromere. Consequently, large inversions lead to increased crossing over and less deletion (more duplication) and an increased risk of abnormal liveborn offspring whereas smaller inversions provide a lower incidence of chiasma formation but, when formed, gametes which are much more unbalanced; all of which translates into very early non-viable conceptions or karyotypically normal gestations such that no reproductive aberrations are noticed.

Until recently, chromosomal analysis of first trimester spontaneous abortions or products of conception has not been uniformly recom-


$\mathrm{p}^{+} \mathrm{q}^{-} \quad$ Inversion

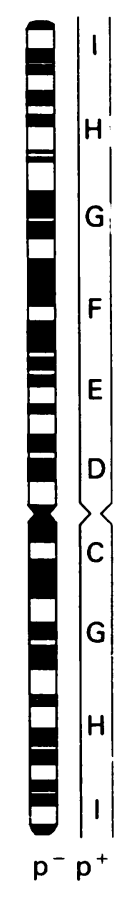

Figure 2 Chromosome 4 in possible paternal gametes. 
mended, ${ }^{18}$ primarily because tissue culture in such cases had a high failure rate, and because contamination with maternal cells frequently distorted the results. Recent advancements in chorionic villus culture has provided dramatic improvements resulting in 99 to $100 \%$ success rates for karyotyping abortus specimens, ${ }^{1920}$ and it would now seem economically expedient to make a concerted effort to karotype all products of conception resulting from a second successive first trimester loss. The knowledge gained can have a major impact on the further evaluation of the couple by indicating a parental abnormality (as in the current case) or, much more frequently, confirming a lethal trisomy or monosomy $\mathrm{X}$ unrelated to, and not requiring delineation of, maternal or paternal karyotype or other maternal disorders. Such information is invaluable for parental counselling and for emotional support.

We thank Dr Richkind for her expert advice.

1 Warburton D, Strobino B. Recurrent spontaneous abortion. In: Bennett MJ, Edmonds DK eds. Spontaneous and recurrent abortion. Boston: Blackwell Scientific Publications, 1987:193-213.

2 De Braekeleer M, Dao TN. Cytogenetic studies in couples experiencing repeated pregnancy losses. Hum Reprod 1990;5:519-28.

3 Bourrouillou G, Colombies P, Dastugue N. Chromosome studies in 2136 couples with spontaneous abortions. Hum Genet 1986;74:399-401.

4 Makino R, Tabuchi T, Nakada K, Iwasaki K, Tamura S, Iizuka $\mathbf{R}$. Chromosomal analysis in Japanese couples with repeated spontaneous abortions. Int $\mathcal{f}$ Fertil 1990;35:26670.

5 Portnoi MF, Joye N, Van Den Akker J, Morlier G, Taillemite JL. Karyotypes of 1142 couples with recurren abortion. Obstet Gynecol 1988; 72:31-4.

6 Simpson JL, Meyers CM, Martin AO, Elias S, Ober C.
Translocations are infrequent among couples having repeated spontaneous abortions but no other abnormal pregnancies. Fertil Steril 1989;51:811-14.

7 Kleczkowska A, Fryns JP, Van den Berghe H. Pericentric inversions in man: personal experience and review of the literature. Hum Genet 1987;75:333-8.

8 Daniel A, Hook EB, Wulf G. Risks of unbalanced progeny at amniocentesis to carriers of chromosome rearrangement: data from United States and Canadian laboratories. Am $\mathcal{F}$ Med Genet 1989;31:14-53.

9 Bensen JT, Nelson LH, Pettenati MJ, et al. First report of management and outcome of pregnancies associated with hereditary orotic aciduria. Am $\mathcal{F}$ Med Genet 1991;41:42631.

10 Borgaonkar DS. Chromosomal variation in man: a catalog of chromosomal variants and anomalies. 6th ed. New York: Wiley-Liss, 1991:147-95 (and personal communication).

11 Simpson JL, Bombard A Chromosomal abnormalities in spontaneous abortion: frequency, pathology and genetic counselling. In: Bennett MJ, Dewhurst J, eds. Sponta-
neous and recurrent abortion. Boston: Blackwell Scientific Publications. 1987:51-76.

12 Rivas F, Garcia-Esquivel L, Rivera H, Jimenez ME, Gonzalez RM, Cantu JM. Inv(4)(p16q21). A five-generation pedigree with 24 carriers and no recombinants. Clin Genet 1987;31:97-101.

13 Baccichetti C, Tenconi A, Caufin D, Bortotto A. Study on segregation of the inversion of chromosome 4 (p15.2q11) in two unrelated families. Hum Genet 1982;62:117-20.

14 Lindberg L, Pelto K, Borgstrom GH. Famial pericentric inversion (3)(p12q24). Hum Genet 1992;89:433-6.

15 Daniel A. Structural differences in pericentric inversions. Application to a model of risk of recombinants. Hum Genet 1981;56:321-8.

16 Therman E. Human chromosomes: structure, behavior, and effects. 2nd ed. New York: Springer-Verlag, 1986:213-15.

17 Harnden DG, Klinger HP, eds. An international system for human cytogenetic nomenclature. Published in collaboration with Cytogenet Cell Genet. Basel: Karger, 1985.

18 Dewald GW, Michels VV. Recurrent miscarriages: cytogenetic causes and genetic counseling of affected families. Genetic causes and genetic counseling

19 Sorokin Y, Johnson MP, Uhlmann WR, et al. Postmortem chorionic villus sampling: correlation of cytogenetic and ultrasound findings. Am $\mathcal{f}$ Med Genet 1991;39:314-16.

20 Strom CM, Ginsberg N, Applebaum M, et al. Analyses of 95 first-trimester abortions by chorionic villus sampling and karyotype. $\mathcal{F}$ Assist Reprod Genet 1992;9:458-61. 